\title{
Transnationale Lohnkoordination zur Stabilisierung des Euro? Gab es nicht, gibt es nicht, wird es nicht geben
}

\author{
Martin Höpner • Martin Seeliger
}

Online publiziert: 27. August 2018

(C) Der/die Autor(en) 2018

Zusammenfassung In Währungsunionen stehen Auf- und Abwertungen zur Korrektur heterogener Inflationsentwicklungen nicht mehr zur Verfügung. Zur Vermeidung von Leistungsbilanzungleichgewichten erfordert der Euro daher die transnationale Koordination von Lohnauftrieben. Seit den 1990er Jahren und verstärkt seit Einführung des Euros bekennen sich die Gewerkschaften zu dem Ziel einer solchen Koordination. Diese Bekenntnisse werden trotz des permanenten Scheiterns der Koordinationsbemühungen aufrechterhalten. Ein Rückgriff auf pragmatistische Handlungstheorien öffnet die Augen für den Umstand, dass die Beteiligten die Diskrepanz zwischen Wünschenswertem und Machbarem durch die Entkoppelung von Reden und Handeln lösen und dass die gegenwärtigen Versuche vor allem das Ziel verfolgen, die Möglichkeit effektiver Lohnkoordination für die langfristige Zukunft zu erhalten. Auf kurze bis mittlere Sicht spricht hingegen nichts für die Annahme, dass die Lohnpolitik in der Lage wäre, mittels einer transnationalen Lohnkoordination auf die Beseitigung der im Euro entstandenen Wechselkursverzerrungen hinzuwirken. Dem Euro fehlt daher bis auf Weiteres die entscheidende Voraussetzung eines friktionsarmen Funktionierens.

Schlüsselwörter Europäische Integration · Gewerkschaften · Lohnpolitik · Wirtschafts- und Währungsunion · Pragmatistische Handlungstheorie

\footnotetext{
M. Höpner $(\bowtie)$

Max-Planck-Institut für Gesellschaftsforschung (MPIfG)

Paulstr. 3, 50676 Köln, Deutschland

E-Mail: hoepner@mpifg.de

M. Seeliger

Institut für Gesellschaftswissenschaften und Theologie, Europa-Universität Flensburg

Auf dem Campus 1a, 24943 Flensburg, Deutschland

E-Mail: martin.seeliger@uni-flensburg.de
} 


\title{
Stabilizing the Euro by the Means of Transnational Wage Coordination? Did not Exist, Does not Exist, Will not Exist
}

\begin{abstract}
Nominal de- and revaluations are impossible in currency unions. In order to prevent current account imbalances, the euro therefore requires the transnational synchronization of labor cost increases. Since the 1990s and especially since the introduction of the euro, European trade unions have announced their goal of coordinating their wage demands transnationally. Such announcements continue even though wage coordination continually fails. Pragmatist action theory makes us aware that actors solve the discrepancy between the desirable and the doable by de-coupling talk and action and that current attempts mainly aim at keeping the possibility of effective wage coordination a goal for the distant future. In the short and middle run, in contrast, nothing indicates that the distorted real exchange rates from which the eurozone suffers could be corrected by the means of wage coordination. The euro therefore lacks the crucial precondition for low-friction functioning.
\end{abstract}

Keywords European integration · Trade unions · Wage policy · Economic and monetary union · Pragmatist action theory

\section{Lohnkoordination als funktionales Erfordernis fester Wechselkursregime}

Mit dem Eintritt in den Euro hat sich der funktionale Druck auf die Lohnpolitik, Beiträge zur transnationalen wirtschaftlichen Stabilisierung zu leisten, fundamental erhöht. Denn eine Währungsunion ist die glaubwürdigste Form eines festen Wechselkursregimes. Unterschiede zwischen den Inflationsdynamiken der beteiligten Länder können nicht mehr durch Auf- und Abwertungen korrigiert werden und tragen, wenn sie sich mittelfristig verfestigen, zur Entstehung und Persistenz von Leistungsbilanzungleichgewichten bei. ${ }^{1}$ Die Fähigkeit zur mittelfristigen transnationalen Synchronisation von Inflationsraten ist daher sowohl Erfordernis als auch Sollbruchstelle fester Wechselkursregime. Da vor dem Hintergrund einer einheitlichen Geldpolitik die Lohnstückkosteninflation entscheidende Determinante der Preisinflation ist, ${ }^{2}$ müssen also die Tarifpartner ihre Lohnpolitik dahin gehend aufeinander abstimmen, dass sie ähnliche Inflationsraten hervorbringen. ${ }^{3}$

\footnotetext{
1 Allerdings verbieten sich monokausale Erklärungen von Leistungsbilanzungleichgewichten. Sie werden von heterogenen Nachfrageentwicklungen mindestens ebenso stark beeinflusst wie von verzerrten Preisen. Besonders plastisch zeigt sich dieser Umstand anhand der jüngst zu beobachtenden Entspannung der Leistungsbilanzen der südeuropäischen Krisenländer, in denen die Binnennachfragen kollabierten. Vergleiche zu den Wirkungen von Preisen auf die Leistungsbilanzen etwa Sinn (2014, Kap. 4), Flassbeck und Lapavitsas (2015) und International Monetary Fund (2015, Kap. 3); zu den Wirkungen von heterogenen Nachfrageentwicklungen auf die Leistungsbilanzen Storm und Naastepad (2015) und Wyplosz (2013).

2 Siehe zum engen Zusammenhang zwischen Lohn- und Preisinflation allgemein Ghali (1999) und speziell mit Bezug auf die Eurozone Collignon (2009, S. 430-431), Flassbeck und Lapavitsas (2015, Kap. 2) und Höpner und Lutter (2018).

3 Vgl. auch Johnston und Regan (2014), die zeigen, dass es von der Beweglichkeit der nominalen Wechselkurse abhängt, ob und wie stark sich Inflationsdifferenziale in Leistungsbilanzungleichgewichte übersetzen.
} 
Ein Problem ist das Fehlen formaler Koordinierungsmechanismen ${ }^{4}$ freilich nur, wenn sich das Ergebnis homogener Lohnauftriebe nicht von selbst einstellt. Wäre die Eurozone ein einheitlicher Konjunkturraum mit zudem ähnlichen Institutionen und Praktiken der Lohnaushandlung, könnte sich der Lohndruck im Prinzip auch in Abwesenheit von zur Koordinierung geschaffenen Institutionen und Prozeduren vereinheitlichen. Aber keine der genannten Bedingungen ist im Euroraum erfüllt. Während der ersten zehn Eurojahre bis zum Eintritt in die Krise wuchsen Italien kumuliert um $13,4 \%$ und Portugal um 17,0\%, Spanien aber um 40,4\%, Griechenland um 43,6\% und Irland sogar um 68,8\% (Daten: OECD). Die Strukturen der Volkswirtschaften der Euro-Teilnehmer unterscheiden sich entlang einer Vielzahl von Dimensionen, wie etwa der Größe ihrer Exportsektoren. Sie verfügen über höchst unterschiedliche Sozialsysteme und gehören, in der Terminologie der Vergleichenden Politischen Ökonomie, unterschiedlichen growth regimes und unterschiedlichen varieties of capitalism an (Esping-Andersen 1990; Baccaro und Pontusson 2016; Hall 2014). Und namentlich unterscheiden sich auch die Modalitäten der Lohnfindung in der Eurozone ganz erheblich, etwa hinsichtlich der Verbreitung von Flächentarifverträgen und Allgemeinverbindlichkeitserklärungen (Hancké 2013; Höpner und Lutter 2018).

Diese Heterogenität lässt die Entstehung eines unkoordinierten Gleichklangs der Lohn- und Preisauftriebe der Euroländer unwahrscheinlich erscheinen und legt die Notwendigkeit von Koordinierungsmechanismen nahe. Gleichzeitig erschwert sie aber auch die Errichtung solcher Mechanismen. Keine Kennerin und kein Kenner der europäischen Arbeitsbeziehungen würde erwarten, dass sich die europäischen Dachverbände der Gewerkschaften in die Lage versetzen, sich Jahr für Jahr auf Lohnleitlinien zu verständigen, die dann 1:1 auf nationaler Ebene durchgesetzt werden. Dass transnationale Lohnkoordination in diesem Maximalsinn nicht existiert und auch nicht bevorsteht, ist schon deshalb trivial, weil die Gewerkschaften selbst unter günstigsten Rahmenbedingungen allenfalls Lohnforderungen abstimmen könnten.

Ein weniger anspruchsvolles, dafür realistischeres Szenario würde die wechselseitige Verpflichtung der Gewerkschaften auf die mittelfristige ${ }^{5}$ Orientierung an einer Lohnregel vorsehen und die regelmäßige Evaluation der Ergebnisse. Über Sanktionsmechanismen gegen ,falsche“ Lohnpolitik würde eine solche Form der Lohnkoordination freilich nicht verfügen. Sie wäre auf den Willen der Beteiligten angewiesen, an der Synchronisation der Lohnauftriebe dauerhaft mitzuwirken, sowie auf die institutionelle und organisatorische Fähigkeit, den aus der Lohnregel resultierenden Erfordernissen auch mittelfristig Rechnung zu tragen. Wie ist es um eine solche Lohnkoordinierung im Euroraum bestellt? Nachfolgend werden wir diese Frage aus unterschiedlichen Perspektiven diskutieren.

\footnotetext{
4 An den Stellen, an denen es einen Unterschied macht, sprechen wir in diesem Beitrag von ,Koordination“, wenn wir auf das Ziel abgestimmter Lohnauftriebe abstellen, von „Koordinierung“ aber, wenn es um das entsprechende gewerkschaftliche Handlungsfeld geht.

5 Kurzfristige Abweichungen wären dann stets zu erwarten und nicht zu beanstanden; worauf es ankäme, wäre die Synchronisation der mittelfristigen Pfade, auf denen sich die nationalen Lohnpolitiken bewegen.
} 
Im Einzelnen werden wir wie folgt vorgehen: In Abschn. 2 werden wir aufzeigen, dass es Initiativen für eine europäische Lohnkoordinierung schon vor dem Euro gab und dass seit der Vorbereitungsphase auf den Euro von einem verfestigten Handlungsfeld europäischer Lohnkoordinierung gesprochen werden kann. In Abschn. 3 stellen wir dar, dass diese Koordinierungsversuche von einer Forschungsliteratur mit verhalten optimistischem Tenor begleitet wurden. Im nachfolgenden Abschn. 4 wenden wir uns dem Problem anhand gesamtwirtschaftlicher Daten zu elf Euro-Ländern für die Jahre 1999 bis 2015 zu. Diese Daten illustrieren, dass im betrachteten Zeitraum keine effektive Lohnkoordination stattgefunden haben kann.

Wie lässt sich die Diskrepanz zwischen der langjährigen Fortexistenz eines Handlungsfelds der Lohnkoordinierung und ausbleibender effektiver Lohnkoordination interpretieren? In Abschn. 5 wenden wir uns einem sektoralen Beispiel zu und zeigen anhand des Verhältnisses der IG Metall zu den Lohnkoordinierungsversuchen des europäischen Dachverbands IndustriALL European Trade Union (im Folgenden: IndustriALL) ${ }^{6}$, dass die Akteure die kurzfristige Unvereinbarkeit der an sie gerichteten Umweltanforderungen durch eine Entkoppelung von Reden und Handeln auflösen. Dieses Ergebnis mag auf Grundlage eines rationalistischen Handlungsmodells kurios erscheinen - ein Eindruck, der aber, wie wir in Abschn. 6 zeigen, verschwindet, wenn man statt des rationalistischen Modells ein soziologisch-pragmatistisches Modell zugrunde legt.

Europäische Lohnkoordination, so unser Fazit in Abschn. 7, dient derzeit und in absehbarer Zukunft der Aufrechterhaltung eines Settings, in dem effektive Lohnkoordination unter deutlich weniger widrigen Umständen in einer ferneren Zukunft stattfinden könnte. Auf kurze bis mittlere Sicht wäre es aber ein Fehler, von der Existenz des Handlungsfelds der Lohnkoordinierung auf die Möglichkeit effektiver europäischer Lohnkoordination zu schließen. Der pragmatische Zweckoptimismus der beteiligten Akteure darf also nicht mit hier und heute verfügbaren Handlungsoptionen verwechselt werden. Auf absehbare Zukunft fehlt dem Euro damit die entscheidende Voraussetzung eines friktionsarmen Funktionierens.

\section{Gewerkschaftliche Lohnkoordinierung in Europa - ein Überblick}

Die Diskussion um Möglichkeit und Notwendigkeit grenzüberschreitender Lohnkoordinierung reicht unter europäischen Gewerkschaften bereits bis in die 1960er Jahre zurück (Pen 1963). Sie ist also älter als der Euro und überdauerte die gesamte Bandbreite der europäischen Wechselkurs- und Währungsregime seit dem Zweiten Weltkrieg: das Bretton-Woods-Regime, die Währungsschlange, das Europäische Währungssystem (EWS) und schließlich die Wirtschafts- und Währungsunion. In den 1970er Jahren mündeten die Diskussionen unter dem Eindruck zunehmender Standortkonkurrenz in erste Initiativen, als Gewerkschaften aus dem Metallsektor entschieden, neben informellem Informationsaustausch auch Abstimmungsversuche

\footnotetext{
6 Dieser Verband schloss sich am 16. Mai 2012 aus der European Metalworkers' Federation, der European Mine, Chemical and Energy Workers' Federation sowie der European Trade Union Federation - Textiles Clothing and Leather zusammen.
} 
zu etablieren (Glassner und Pochet 2011, S. 12). Das Ziel dieser Initiativen bestand in der Verhinderung von lohnpolitischer Unterbietungskonkurrenz. In schriftlich fixierte Lohnregeln mündeten die Initiativen in diesem Stadium nicht.

Mit den Beschlüssen zur Errichtung einer Wirtschafts- und Währungsunion erlangten diese Initiativen in den frühen 1990er Jahren neuen Nachdruck, was darauf hindeutet, dass die zentrale Stellung der Lohnpolitik in Währungsunionen von den damaligen Akteuren erkannt wurde. Zwar war es auch in „,diskretionären“ Wechselkursregimen wie dem EWS wünschenswert, es gar nicht erst zu unterschiedlichen Raten der Lohn- und Preisinflation kommen zu lassen. Wo solche Divergenzen aber auftraten, konnten sie, wie im EWS auch häufig geschehen, durch Auf- und Abwertungen korrigiert werden (siehe die Einzelheiten in Höpner und Spielau 2016). In der Währungsunion hingegen mussten sich heterogene Lohnentwicklungen, sofern sie auf die Preise durchschlugen, direkt in Wettbewerbsvorteile oder -nachteile übersetzen. Auf Initiative des Europäischen Metallgewerkschaftsbunds (EMB) entwickelten Repräsentanten der vertretenen Gewerkschaften im Laufe der 1990er Jahre die sogenannte Koordinierungsregel (Schroeder und Weinert 2003, S. 578). Sie besagte, dass nominale Lohnauftriebe mindestens der Summe aus Produktivitätszuwächsen und Inflation entsprechen sollen.

$\mathrm{Zu}$ beachten ist an dieser Stelle, dass die Regel mehrere Interpretationsmöglichkeiten zulässt. Mit den Produktivitätszuwächsen sind die jeweiligen Prognosen für die nationalen, gesamtwirtschaftlichen Produktivitätszuwächse des betreffenden Jahres gemeint (jedenfalls ist uns keine anderslautende Interpretation bekannt). Das Problem ist der Verweis auf die Inflation. Ist damit die nationale Inflationsrate gemeint, verfehlt die Regel unter der Randbedingung fester Wechselkurse ihr etwaiges Ziel der Verhinderung von transnationalen Ungleichgewichten. Denn die Orientierung an einer solchen Lohnregel würde dazu führen, dass jedes beteiligte Land seine aktuelle Inflationsrate verstetigt - erreicht würde also genau das, was in einer Währungsunion nicht eintreten darf, weil sich unterschiedliche Preisentwicklungen nicht mehr durch Wechselkursanpassungen korrigieren lassen.

Funktional im Sinne der Verhinderung von Außenhandelsungleichgewichten wäre daher die Orientierung an der Durchschnittsinflationsrate des Euroraums oder an der Zielinflationsrate der EZB. Fest steht, dass das Ziel der Stabilisierung des Euroraums nach und nach in das Setting einwanderte. In einem im Jahr 2012 vom Exekutivausschuss von IndustriALL verabschiedeten Grundsatzpapier wird als Ziel der Lohnkoordination explizit auf die Verhinderung von „Ungleichgewichten im Außenhandel“ verwiesen. Auch die IG Metall verweist in ihrem 2009 veröffentlichten Europapolitischen Memorandum im Unterabschnitt „Europäische Tarifpolitik“ auf die speziellen Erfordernisse einheitlicher Währungsräume hin (IG Metall 2009, Abschn. 4.2); seit der Tarifrunde 2013 begründet die IG Metall Lohnforderungen zudem explizit unter Bezugnahme auf die Zielinflationsrate der EZB.

Die Verabschiedung der Koordinationsregel durch den EMB markierte nicht nur in Bezug auf ihre nominelle Programmatik einen Wendepunkt. Während die tarifpolitische Zusammenarbeit auf europäischer Ebene bis dahin vor allem in das Ressort der internationalen Sekretäre der beteiligten Gewerkschaften fiel, nahm sich nun ein „Tarifpolitischer Ausschuss“ des Themas an. Hier traten neben einem Mitarbeiter des EMB auch Vertreter der Metallgewerkschaften aus Deutschland, Österreich, 
Schweden und Belgien zusammen. Wie im Jahr 1996 verbindlich auf einer Sitzung in Frankfurt a. M. beschlossen, sollten die beteiligten Organisationen die vereinbarte Regel in ihren lokalen Tarifverhandlungen umsetzen, um dem im gemeinsamen Markt verstärkten Standortwettbewerb zu begegnen (Perez 2002).

Um die Koordinierung von Lohnabschlüssen im Sinne dieser Regel zu unterstützen, etablierten die europäischen Metallgewerkschaften mit dem Eucob@n-System eine Datenbank, die einen Überblick über das Tarifgeschehen in den Mitgliedsländern ermöglichen sollte. Neben den Spezifika der nationalen Lohnfindungssysteme ging es hierbei vor allem um die Ergebnisse der lokalen Tarifrunden (Schulten 2004, S. 292). Zusätzlich zu diesem Monitoring sah die Beschlusslage wechselseitige Besuche nationaler Delegierter bei den Tarifverhandlungen ausländischer Schwesterorganisationen sowie die Abhaltung großer tarifpolitischer Konferenzen auf europäischer Ebene vor. Die Abstimmung folgt also einem Muster ,weicher Koordinierung": Sanktionsmöglichkeiten, die die europäischen Verbände gegenüber den nationalen Mitgliedsorganisationen anwenden könnten, bestehen allenfalls im Sinne eines weichen ,naming and shaming“.

\section{Gewerkschaftliche Lohnkoordinierung im Spiegel der Forschung}

Aus Sicht der Gewerkschaften bewegen sich die Zielgrößen der Lohnpolitik im Spannungsfeld zweier Bezugspunkte, den Arbeitsplatz- und Verteilungsinteressen der Verbandsmitglieder einerseits und den durch die Lohnpolitik herbeigeführten makroökonomischen Ergebnissen andererseits (Bispinck und Schulten 2006, S. 254). Wir haben bereits gesehen, dass sich die Notwendigkeit, neben den unmittelbaren Verbandsinteressen bei der Wahl von Lohnforderungen auch makroökonomische Gesichtspunkte zu berücksichtigen, mit dem Eintritt in feste Wechselkurse um eine transnationale Dimension erweitert hat. In den Exportsektoren lassen sich für die Gewerkschaften nunmehr zwei Möglichkeiten der strategischen Orientierung unterscheiden. Um ihren Mitgliedern Arbeitsplatzsicherheit zu garantieren, können sie einerseits versuchen, die national-sektorale Wettbewerbsfähigkeit durch - im Vergleich mit den ausländischen Nachbargewerkschaften - niedrige Lohnforderungen zu gewährleisten und damit reale Abwertungen herbeizuführen, die sich anschlieBend nicht mehr durch Wechselkursanpassungen korrigieren lassen. Andererseits, und dies im Sinne der hier betrachteten Abstimmungsinitiativen, können sie eine koordinierte Lohnentwicklung anstreben, die reale Abwertungswettläufe verhindert.

Die begleitende empirische Forschungsliteratur zur Lohnkoordinierung (beispielsweise Johnston 2009; Traxler et al. 2008; Traxler und Brandl 2009) sowie auch die eher politischen und konzeptionellen Beiträge (Sterkel et al. 2004; Schulten 2001; Pulignano 2010) stellen dieses Spannungsfeld in Rechnung, das sich auch als ein Auseinanderdriften von aus internationaler Perspektive Wünschbarem und angesichts der Mitgliederlogik Machbarem beschreiben lässt. Einerseits wäre eine transnational abgestimmte Lohnpolitik zur Verhinderung von Lohnunterbietungskonkurrenz und speziell vor dem Hintergrund fester Wechselkurse zur Verhinderung von Leistungsbilanzungleichgewichten wünschenswert. Andererseits setzen Vorbehalte gegen die Abgabe lohnpolitischer Kompetenzen an übergeordnete 
Ebenen, etwaige Anreize zum Eintritt in Lohnunterbietungskonkurrenz, die über die beteiligten Länder und Sektoren ungleich verteilte Durchsetzungsfähigkeit der Gewerkschaften und die Heterogenität der in der Eurozone vertretenen Tarifsysteme der Machbarkeit effektiver Koordination Grenzen.

Auffällig ist nun, dass die Forschungsliteratur die Fähigkeit zur Auflösung des Spannungsfelds zwischen Wünschbarem und Machbarem mit deutlich optimistischem Einschlag behandelt. Besonders klar manifestiert sich dieser Einschlag in der Frühzeit der Lohnkoordinierung und der sie begleitenden Forschung. So schließen etwa Hoffmann und Hoffmann (1997, S. 130), die europäischen Gewerkschaften seien ,not far from a convergent and coordinated wage strategy and a coordination of national negotiations, which would be a necessary and appropriate answer to the processes of Europeanization and globalization“. Ähnlich diagnostiziert Weinert (2001, S. 333) auch nach dem Eintritt in die Währungsunion einen „Prozess schleichender Entdiplomatisierung" in diesem bislang unverbindlich koordinierten Politikbereich. Ganz allgemein lassen sich in den Beiträgen mit der Wahrnehmung von Internationalisierung als Funktionserfordernis (Glassner und Pochet 2011) und solidarischem Wertbezug (Schulten und Müller 2013) zwei Referenzgrößen als treibende Kräfte dieser Entwicklung ausmachen.

Als favorisierte Ebene für Lohnkoordinierung führen die in erster Linie konzeptionell orientierten Beiträge die sektorale Arena an. Hier, so etwa Traxler (2000, S. 106), könnten die ,im Entstehen begriffenen, weichen', netzwerkförmigen Institutionen" unter bestimmten Voraussetzungen für eine effektive Koordination ausreichen. Lohnkoordination sei daher, so Traxler (2000, S. 106) weiter, ,,auf der Grundlage nicht-hierarchischer, netzförmiger Koordinierungsmechanismen" möglich. Für die Abstimmung von Tarifverhandlungen innerhalb des gleichen Sektors spreche weiterhin die Tatsache, dass Interessen von Gewerkschaften aus dem gleichen Wirtschaftssegment leichter in Einklang zu bringen seien (Glassner 2009, S. 147). Zusätzlich zu wirtschaftlichen Beweggründen weisen die Beiträge zum Thema aber auch auf die Bedeutung kultureller Gemeinsamkeiten und geteilter Wertbezüge hin. So betonen etwa Glassner und Pernicka (2014b) bei ihrer Sekundäranalyse aller tarifpolitischen Koordinierungsinitiativen nationaler und europäischer Industrie- und Metallgewerkschaften das Vorhandensein kulturell-kognitiver Institutionen, die im Prozess internationaler Zusammenarbeit geteilte Wertbezüge vermitteln (zum Beispiel durch den bei tarifpolitischen Konferenzen erfolgenden Austausch mit anderen Delegierten).

Verfolgt man die begleitende Forschungsliteratur weiter im Zeitverlauf, erkennt man eine abnehmende Intensität des Optimismus (siehe zur optimistischen Tendenz der frühen Beiträge auch Schulten und Bispinck 1999; Schroeder und Weinert 2003, 2004; Marginson und Traxler 2005). Einerseits blieben die angesichts der EuroGründung prognostizierten oder zumindest erhofften Durchbrüche aus, andererseits verdeutlichte der Eintritt in die Eurokrise in den Jahren ab 2009 die Schwere der Probleme, die durch das Ausbleiben effektiver Lohnkoordination ausgelöst wurden (Erne 2008, 2013; Sterkel und Wiedemuth 2011; vgl. zu den Resultaten der Koor- 
dinierungsversuche den nachfolgenden Abschn. 4). ${ }^{7}$ Eine positive Darstellungsform kommt, so zeigt etwa Henning (2013, S. 163), häufig zum Preis einer Relativierung der nominellen Zielsetzung zustande. Aus seiner Sicht verfolgt der Europäische Metallgewerkschaftsbund in seinen Abstimmungsinitiativen lediglich einen weichen Koordinierungsanspruch, dessen Verbindlichkeitsgrad sich aus einer ,moralischen Selbstverpflichtung“ ableite (Henning 2013, S. 163). Als mittel- oder langfristiges Ziel dieser Abstimmungsaktivitäten im Rahmen ,transnationaler Kommunikationsräume“ (Henning 2013, S. 163) ist die Strategie explizit auf die ferne Zukunft hin orientiert (wir werden auf diesen Umstand ausführlich zurückkommen):

Hinter diesem regionalen gewerkschaftlichen Vernetzungsansatz steht die (erfahrungsgesättigte) Überlegung, dass sich die Europäisierung der Tarifpolitik letztlich nur als Ergebnis eines Prozesses der zunehmenden gegenseitigen Kommunikation, Vertrauensbildung und Verständigung der nationalen Tarifakteure herstellen wird und dass es wichtig ist, die dazu erforderlichen strukturellen Voraussetzungen zu schaffen (Henning 2013, S. 274).

Während optimistische Vertreter vor diesem Hintergrund zunächst immer wieder teleologische Einschätzungen geäußert hatten - so verweisen etwa Glassner und Pochet (2011, S. 8) auf ,,an emerging (but still very fragmented) EU framework of industrial relations" - wurden diese zuletzt auch relativiert:

When it comes to assess these - indeed patchy and polycentric - processes of horizontal Europeanization for their potential to contribute to the emergence of a European field of wage policy, there is doubt that transnational pattern bargaining based on common wage norms will spill over to other regions and sectors (Glassner und Pernicka 2014a, S. 14).

Insgesamt also zeigt der Forschungsstand eine zwar im Zeitverlauf nachlassende, insgesamt aber gleichwohl deutliche Tendenz, die Frage nach der Existenz von Lohnkoordination zu bejahen oder die Koordination zumindest als im Entstehen begriffen zu kennzeichnen.

\section{Die Unterschiedlichkeit der Lohnauftriebe im Euroraum}

Der bis hier referierte Stand der Dinge lässt keine Zweifel daran offen, dass spätestens seit der Vorbereitungsphase auf den Euro ein gewerkschaftliches Handlungsfeld transnationaler Lohnkoordinierung existiert. $\mathrm{Ob}$ in diesem Handlungsfeld aber tatsächlich effektive Lohnkoordination stattgefunden hat, lässt sich nur über einen Vergleich der Lohnauftriebe im Euroraum entscheiden, dem wir uns nun zuwenden wollen.

Tabelle 1 informiert über die Entwicklung der nominalen Lohnstückkosten für elf der zwölf Gründungsländer des Euro (ohne Luxemburg) in zwei Zeiträumen, nämlich erstens den ersten zehn Eurojahren zwischen 1999 (dem Jahr der unwider-

\footnotetext{
7 Siehe zum nachlassenden Optimismus etwa auch Glassner und Watt (2010), Lehndorff (2014), Marginson (2014) und Marginson et al. (2014).
} 


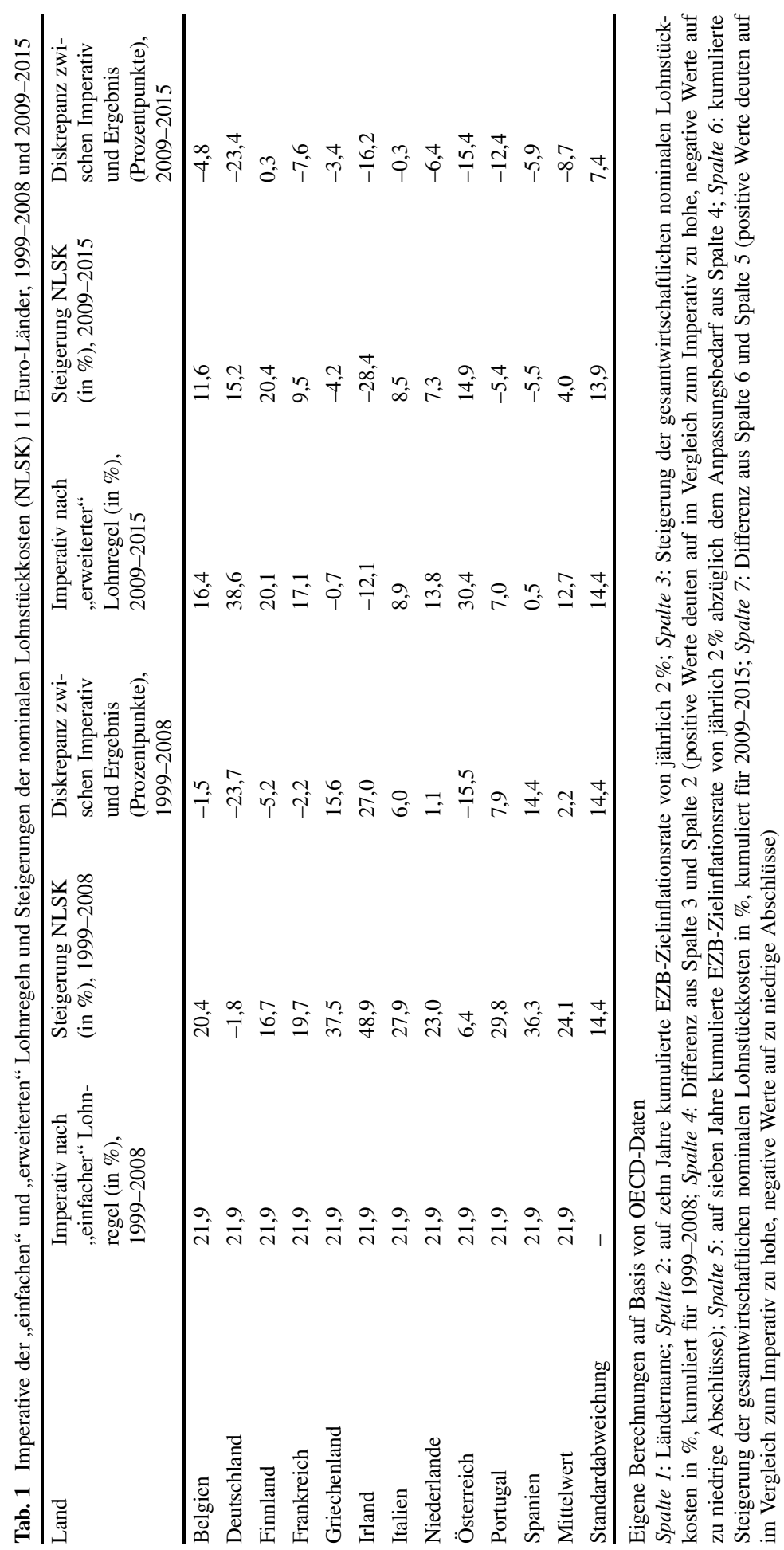


ruflichen Fixierung der Wechselkurse) und 2008 (dem letzten Jahr vor Ausbruch der Finanz- und Eurokrise) und zweitens den Krisenjahren 2009 bis $2015 .{ }^{8}$ Konzentrieren wir uns zunächst auf die ersten drei Spalten nach den Ländernamen (Spalten 2-4). Gegen welche Zielgröße wären die lohnpolitischen Ergebnisse zu prüfen? Stellt man in Rechnung, dass Nominallohnsteigerungen hinsichtlich ihrer Wirkung auf das Preisniveau neutral sind, wenn sie sich im Einklang mit der Produktivitätsentwicklung befinden (gleichbedeutend mit konstanten Lohnstückkosten), und berücksichtigt zudem das Inflationsziel der EZB in Höhe von $2 \%$, dann ergibt sich als ,goldene“ oder ,einfache“ Lohnregel für die ersten zehn Eurojahre eine Zielgröße von 21,9\% (das sind jährliche 2\%, auf zehn Jahre aufkumuliert - Spalte 2). ${ }^{9}$ Die Spalten 2 und 3 verdeutlichen, dass die Orientierung an dieser Lohnregel im Länderdurchschnitt nicht schlecht funktioniert hat. Der Durchschnitt von 24,1\% an nominalen Steigerungen der Lohnstückkosten (Spalte 3) ist nur 2,2 Prozentpunkte (Spalte 4) von der Zielgröße entfernt. Angesichts des recht langen Betrachtungszeitraums von zehn Jahren ist das keine große Abweichung.

Allerdings ist die Varianz um den Durchschnitt enorm: Um durchschnittlich ganze 14,4 Prozentpunkte streute der durchschnittliche Auftrieb der Lohnstückkosten um den Wert von 24,1\%. Zwischen dem Land mit den höchsten (Irland) und den niedrigsten (Deutschland) Lohnauftrieben finden wir sogar eine Spannweite von um die 50 Prozentpunkten vor. Die in Tab. 1 gewählte Darstellungsweise illustriert eindrucksvoll, was im Euroraum bis zum Ausbruch der Eurokrise geschah: Nur in recht wenigen Ländern - Belgien, Frankreich, Niederlande - stiegen die Lohnstückkosten in ungefährer Entsprechung der Vorgaben der ,einfachen Lohnregel“". In einer zweiten Ländergruppe schossen die Löhne deutlich über. Hierzu zählen Griechenland, Spanien und Irland und mit Abstrichen auch Italien und Portugal. Deutschland, Österreich und zudem auch Finnland betrieben hingegen Lohnzurückhaltung, wobei Deutschland aufgrund seiner sogar sinkenden nominalen Lohnstückkosten eine besonders auffällige Positionierung einnimmt. Wenn es während der ersten zehn Eurojahre Bestrebungen zur effektiven transnationalen Koordination der Lohnauftriebe im Euroraum gegeben hat, dann sind diese offenbar gescheitert.

Mit dem Eintritt in die Eurokrise sind die an die Lohnpolitik gerichteten Imperative noch herausfordernder geworden. Worauf hätte eine transnationale Lohnkoordination nunmehr hinwirken müssen? Die Orientierung an der „einfachen Lohnregel“, der zufolge die Lohnstückkosten in allen beteiligten Ländern um jährlich $2 \%$ hätten steigen müssen, konnte nicht mehr genügen, musste es angesichts der Unmöglichkeit nominaler Auf- und Abwertungen nun doch darüber hinaus darum gehen, die durch die auseinanderlaufenden Lohnauftriebe der ersten zehn Eurojahre entstandenen Wechselkursverzerrungen wieder zu bereinigen. Spalte 5 gibt Aufschluss über die Zielgrößen, gegen die die Lohnauftriebe der Jahre 2009 bis 2015 geprüft werden müssen. Die dargestellten Daten ergeben sich aus der Zielinflationsrate der

\footnotetext{
8 Weil die verfügbaren Daten für das Jahr 2016 zum Zeitpunkt der Abfassung dieses Beitrags (im Frühsommer 2017) noch Lücken aufwiesen, endet die Betrachtung mit dem Jahr 2015.

${ }^{9}$ Da dieser Umstand regelmäßig für Verwirrung sorgt: Die Diskrepanz zwischen 20,0 und 21,9\% ergibt sich aus der für die Kumulation von Steigerungsraten notwendigen Berücksichtigung des Zinseszinseffekts.
} 
EZB (14,9\%, das ist die auf sieben Jahre kumulierte Zielinflation) unter zusätzlicher Berücksichtigung der in Spalte 4 gezeigten Verwerfungen aus den ersten zehn Eurojahren. Diese „erweiterte Lohnregel“ hätte also beispielsweise im Fall Portugals nicht nur lediglich 14,9\% an Lohnstückkostensteigerungen verlangt, sondern zusätzlich eine Korrektur nach unten in Größenordnung des „Überschießens“ aus der Vorperiode (das waren 7,9\%), woraus sich insgesamt ein lohnpolitischer Imperativ von 7,0\% ergibt. Auf den ersten Blick erkennbar ist der außerordentlich hohe Wert für Deutschland: Um beachtliche 38,6\% hätten die nominalen Lohnstückkosten Deutschlands in den betreffenden Jahren steigen müssen, hätte es eine transnationale Lohnkoordination gegeben, die sichergestellt hätte, dass Deutschland neben der Orientierung an der EZB-Zielinflation jene Wechselkursverzerrungen korrigiert, die es selbst zu verantworten hat.

Was aber ist tatsächlich geschehen? Anders als während der ersten zehn Eurojahre wurden die Imperative der Lohnregel nunmehr auch im Durchschnitt aller Länder verfehlt. Anstelle der geforderten durchschnittlich 12,7\% stiegen die nominalen Lohnstückkosten in der gezeigten Ländergruppe lediglich um 4\%, was konkret bedeutet, dass von der Lohnpolitik deflationäre Impulse ausgingen. Noch bemerkenswerter aber ist, dass die Varianz um diesen Durchschnitt der Unterschiedlichkeit der lohnpolitischen Ergebnisse der ersten zehn Eurojahre in nichts nachsteht: Die Länderdaten streuen im Schnitt um 13,9 Prozentpunkte um den Durchschnitt der Steigerungsraten von $4 \%$. Leicht zu erkennen ist hierbei ein Muster: Alle Krisenländer disinflationierten - vor allem natürlich unter dem Druck der Troika - sogar mehr als gefordert.

Die Länder aber, die in der Vorperiode Lohnzurückhaltung betrieben hatten, kamen den Krisenländern nicht durch lohnpolitische Inflationierung entgegen. Das gilt namentlich für Deutschland und Österreich, die lediglich Ergebnisse hervorbrachten, die ungefähr den Imperativen der ,einfachen“ Lohnregel entsprachen. Das bedeutet konkret, dass diese Länder die Krisenländer mit der gesamten lohnbezogenen Anpassungslast allein ließen und maßgeblich dafür verantwortlich zu machen sind, dass die Eurozone dem Ziel einer Korrektur der realen Wechselkursverzerrungen nach Ablauf des betrachteten Zeitraums noch nicht spürbar nähergekommen ist. Man kann das auch wie folgt ausdrücken: Im krassen Gegensatz zur in Deutschland kommunizierten Problemdeutung kranken die innereuropäischen realen Wechselkurse nicht mehr am Fehlverhalten der südeuropäischen Euro-Teilnehmer - dieses Fehlverhalten ist inzwischen bereinigt! -, sondern am Verhalten der Länder der ehemaligen „DM-Zone“ mit Deutschland im Kern.

\section{Ein Blick in die Praxis}

Die bisher dargestellten Ergebnisse ergeben ein Puzzle im besten Wortsinn: Es ist unklar, wie sich die Einzelteile zu einem kohärenten Ganzen fügen sollen. Offenbar existiert im Euroraum keinerlei effektive Lohnkoordination, trotz ihres permanenten Scheiterns aber weiterhin ein Handlungsfeld namens Lohnkoordinierung. Wie passen diese Befunde zusammen? Nachfolgend werden wir die Blackbox „Lohnkoordinierung" öffnen, um anhand eines besonders exponierten Beispiels in Erfah- 
rung zu bringen, wie die Praktiker mit dem Scheitern der Koordination umgehen. Es geht um die deutsche Tarifvertragspartei IG Metall im Verhältnis zum europäischen Verband IndustriALL. Wir sahen in Abschn. 4, dass sich in keinem anderen Gründungsmitglied der Eurozone, relativ zu den Imperativen der Lohnregel, mehr Anpassungsbedarf aufgetürmt hat als in Deutschland. ${ }^{10}$ Gleichzeitig sind die Koordinierungsinitiativen in den in IndutriALL zusammengefassten Industriesektoren am weitesten fortgeschritten. Der uns hier interessierende Konflikt sollte in dem Fallbeispiel also besonders deutlich zutage treten.

Nachfolgend werden wir uns zunächst den strukturellen Parametern des deutschen Regimes der Lohnfindung zuwenden, in dem sich die an der Lohnkoordinierung Beteiligten bewegen (Abschn. 5.1). Die anschließend in Abschn. 5.2 gezeigten O-Töne entstammen einem Forschungsprojekt über die Entwicklung gemeinsamer politischer Positionen in europäischen Gewerkschaftsorganisationen, in dessen Rahmen neben der Lohnkoordination auch die Konflikte um die Arbeitnehmerentsendung und um die Forderung nach einem europäischen Mindestlohn untersucht wurden (ausführlich: Seeliger 2017). Die Datenbasis zum Teilbereich der Lohnkoordination setzt sich aus insgesamt zwanzig Experteninterviews mit Vertretern unterschiedlicher nationaler Gewerkschaftsorganisationen sowie Vertretern europäischer Dachverbände zusammen.

\subsection{Strukturelle Gesichtspunkte}

In der Literatur über „Spielarten des Kapitalismus“ (Hall und Soskice 2001) gelten Deutschland und insbesondere sein Industriesektor - und dort wiederum zuvörderst die Metall- und Elektroindustrie - als paradigmatischer Fall koordinierter Lohnfindung, was in unserem Zusammenhang konkret bedeutet, dass Deutschland ein vergleichsweise hohes Maß an intentionaler Steuerbarkeit der Lohnentwicklung zugesprochen wird. Weicht nun, wie in Abschn. 4 festgestellt, Deutschland in auffälligem Maße von der Lohnentwicklung der Eurozone ab, mag man vorschnell versucht sein, dieses Ergebnis einem Mangel an Wollen des Lohnführers IG Metall an der Befolgung der Imperative der Lohnkoordination zuzuschreiben statt einem Mangel an Können.

Die Interpretation würde dann ungefähr wie folgt lauten: Die 2,3 Mio. Mitglieder der IG Metall in den Branchen Eisen und Stahl, Handwerk, Holz und Kunststoff, Metall und Elektro sowie Textil sind dem internationalen Wettbewerb in besonderem Maße ausgesetzt, was sich auch auf die Vertretungsarbeit der IG Metall überträgt. In ihrer politischen Orientierung erkennt beispielsweise Rüb (2009, S. 144) eine reformistisch-wirtschaftsdemokratische Ausrichtung, welche die Gewerkschaft im Wege einer Gewährleistung von Wettbewerbsfähigkeit und Produktivitätssteigerung verfolgt. Der damit einhergehende sektorale Korporatismus steht in potenziellem

\footnotetext{
10 In Höpner und Lutter (2018, Tab. 1) wird gezeigt, dass der Befund einer ausgeprägten deutschen Lohnzurückhaltung auch dann Bestand hat, wenn man statt gesamtwirtschaftlicher Daten das verarbeitende Gewerbe betrachtet (das verarbeitende Gewerbe ist der beste zur Verfügung stehende Proxy für den Exportsektor). Zwischen 1999 und 2008 sanken die nominalen Lohnstückkosten dort um 5,0\%. Deutschland ist das einzige Land der zwölf Gründungsmitglieder der Eurozone, in dem die nominalen Lohnstückkosten bis zum Eintritt in die Eurokrise sowohl im verarbeitenden Gewerbe als auch gesamtwirtschaftlich sanken.
} 
Widerspruch zur Einhaltung einer formalen Lohnregel, die den Kampf um relative Wettbewerbsfähigkeit auf der Kostenseite ja gerade begrenzen soll und die sich zudem nur im Konflikt mit dem Sozialpartner durchsetzen lässt (vgl. Abschn. 3). Thelen (2014, S. 36) fasst die Entwicklung der Handlungsorientierung der Organisation wie folgt zusammen: ,Rather than conflict, we observe an intensification of cooperation between labor and capital in the interest of what remains a very competitive export sector" (Thelen 2014, S. 36).

Die Charakterisierung der Arbeitsbeziehungen im deutschen Exportsektor als „wettbewerbskorporatistisch“ ist zweifellos korrekt. Gleichwohl wäre es ein Fehler, hieraus zu folgern, dass Lohnzurückhaltung in einem solchen Umfeld allein dem Wollen zuzuschreiben ist, was logisch zwingend nach sich zieht, dass man auch anders könnte. Die Literatur über den Korporatismus und das „deutsche Modell“ der Arbeitsbeziehungen entstand in den 1970er- bis 1990er Jahren des vergangenen Jahrhunderts (Streeck 1994) und damit in einem Umfeld, in dem lohnpolitische Handlungsfähigkeit stets im Hinblick auf die Fähigkeit diskutiert wurde, eine möglichst stabilitätsorientierte Lohnpolitik betreiben und damit Spielräume für beschäftigungsorientierte Fiskal- und Geldpolitiken öffnen zu können (grundlegend: Scharpf 1987). Dass sich die Akteure des deutschen Lohnregimes offenbar in die Lage versetzten, von ihrer Lohnpolitik besonders niedrige Inflationsimpulse ausgehen zu lassen, impliziert aber nicht, dass sie auch zum Gegenteil in der Lage wären, nämlich zur lohnpolitisch gesteuerten Inflationierung, um die im Euro entstandenen Wechselkursverzerrungen wieder zu beheben.

Um dem möglichen Fehlschluss eines unbegrenzten Optionsraums der deutschen Exportgewerkschaften entgegenzuwirken, expansive Lohnpolitik zu betreiben, sind unseres Erachtens mindestens folgende Gesichtspunkte zu bedenken:11 Während die Exportsektoren vom Mitgliederrückgang der Gewerkschaften weniger betroffen sind als die Dienstleistungssektoren, finden wir in den dem internationalen Wettbewerb ausgesetzten Sektoren einen zunehmenden Transfer tarifpolitischer Kompetenz auf die betriebliche Ebene vor. Von der Einführung neuer Arbeitszeit- und Beschäftigungsmodelle in den 1980er Jahren über die systematische Öffnung von Flächentarifverträgen zu Beginn der 1990er Jahre bis zum Pforzheimer Abkommen ${ }^{12}$ des Jahres 2004 erfolgte eine zunehmende Verbetrieblichung des Klassenkonflikts (Haipeter 2011, S. 31). Auf Betriebs- und Unternehmensebene sehen sich die Interessenvertreter der Beschäftigten einem steten Investitionswettbewerb und zudem der andauernden Drohung mit Arbeitsplatzverlagerungen ausgesetzt, was sich in den Druck übersetzt, den durch die Dezentralisierung der Lohnfindung entstandenen Spielraum auch zu nutzen. Je weiter entfernt von der gesamtwirtschaftlichen Arena die Lohnpolitik also erfolgt, umso größer die Schwierigkeit, diese an gesamtwirtschaftlichen oder gar europäischen Imperativen zu orientieren, wenn diese Orientierung die Arbeitsplatzsicherheit der Kernbelegschaften gefährden könnte.

\footnotetext{
11 Vgl. auch Höpner (2016).

12 Mit der Zustimmung der Tarifparteien können Unternehmensleitungen und Betriebsräte demnach $\mathrm{Ne}-$ gativabweichungen vom vereinbarten Lohn erwirken.
} 
Spätestens seit dem Ende der 1990er Jahre und beschleunigt durch die HartzReformen setzte zudem ein institutioneller Wandel im Verhältnis von Staat, Wirtschaft und Gesellschaft ein (Hassel und Schiller 2010; Trampusch 2009), der sich tarifpolitisch in der Ausweitung des Niedriglohnsektors, einer indirekten Senkung von Lohnkosten durch die Zunahme prekärer Beschäftigungsformen wie Leiharbeit oder Werksverträge sowie einer strukturellen Schwächung der gewerkschaftlichen Verhandlungsposition durch höhere subjektive Kosten von Arbeitslosigkeit niederschlägt. Insgesamt ist also festzuhalten, dass die deutschen Gewerkschaftsspitzen selbst dann, wenn sie etwaiger strategischer Lohnzurückhaltung abschwören und sich glasklar auf das Ziel einer lohnpolitisch herbeigeführten Inflationierung zur Bereinigung der in der Eurozone aufgetürmten Wechselkursverzerrungen verständigen würde, auf ein ungünstiges strukturelles Umfeld stoßen würde.

\subsection{Die Sicht der Praktiker}

Bemerkenswert ist, dass weder die kompetitive Lohnzurückhaltung Deutschlands noch das strukturell ungünstige Umfeld für eine deutlich expansivere Lohnpolitik zu Abstrichen bei dem nominellen Bekenntnis zur transnationalen Lohnkoordinierung führte. Wie die IG Metall in ihrem europapolitischen Memorandum zum Thema verlauten lässt, zielt ein „europapolitisches Mainstreaming“ (IG Metall 2009, Abschn. 5) auf die Etablierung eines europäischen Bewusstseins unter den Mitgliedern, welches ,,auf allen Ebenen der Organisation selbstverständlich werden muss“ (IG Metall 2009, Abschn. 5). Die Initiative europäischer Lohnkoordinierung stellt hierbei, dies belegen eine Reihe von Veröffentlichungen (IG Metall 2005, 2009), den zentralen Bezugspunkt des europapolitischen Engagements der IG Metall dar. Auf diesen besonderen Stellenwert verweist die IG Metall (2009, S. 24) explizit: „Über die Handlungsfähigkeit der Gewerkschaften in Europa wird nicht in Sonntagsreden entschieden“.

Diese Programmatik wird auch von IndustriALL Europe geteilt. Hinsichtlich ihrer tarifpolitischen Orientierung formuliert die Organisation eine Reihe von Zielgrößen, darunter die Einhaltung der Lohnformel, den Ausbau der Koordinierungsinitiativen sowie die Verbesserung des Monitorings ihrer Wirksamkeit (IndustriALL 2012, S. 17). Dass der Dachverband hierbei über keine harten Sanktionsmechanismen verfügt, beschränkt ihn auf die Rolle eines Moderators, der den Mitgliedsgewerkschaften lediglich ein Forum zur Diskussion der Ergebnisse nationaler Tarifrunden bieten kann. Diesem unverbindlichen Charakter zum Trotz betonen die befragten Tarifsekretäre die praktische Relevanz der Koordinierungsregel in fast allen Interviews. Der gemeinsame Bezugsrahmen, so ein Interviewpartner, stelle „keine Guideline“, sondern ,eine Regel“ dar.

Vor dem Hintergrund der Verfehlung dieser Regel sieht sich die IG Metall deutlicher Kritik ausgesetzt. Deppe (2012, S. 94) beispielsweise sieht die deutschen Gewerkschaften in der „Rolle eines ,Juniorpartners“ deutscher Hegemonialpolitik in der EU“. Der Vorwurf intentionaler Lohnzurückhaltung wird auch unter den internationalen Schwestergewerkschaften der IG Metall geäußert, die die zurückhaltenden Tarifforderungen der IG Metall als ,chosen strategy“ beschreiben. Entsprechende Vorwürfe werden durch die Vertreter sowohl der regionalen Tarifabteilungen als 
auch der Repräsentanten der Frankfurter Zentrale der IG Metall entschieden zurückgewiesen.

Alle befragten Tarifsekretäre der IG Metall führen die exzeptionelle deutsche Lohnentwicklung seit Einführung des Euro auf die in Abschn. 5.1 skizzierten Rahmenbedingungen zurück. Die Erfolgsaussichten effektiver Lohnkoordination im engeren Sinne schätzen die tarifpolitischen Vertreter der IG-Metall-Zentrale daher äußerst pessimistisch ein:

Das Problem ist hier einfach, also eins der Grundprobleme ist, bei dieser Lohnkoordinierung. Wie soll das bitte funktionieren? Wir haben kein Rezept dafür und an der Stelle muss man sagen, wir haben es versucht in den letzten Jahren.

Ebenfalls mit dem Hinweis auf die Schwierigkeiten, die einer erfolgreichen Umsetzung im Wege stehen, relativieren die befragten Tarifsekretäre die Relevanz der Regel für die tarifpolitische Praxis. Eine Orientierung an der Regel sei kaum zu vermitteln: „Also, Du kannst Dich beim Warnstreik da nicht hinstellen und sagen, wir machen es deshalb, weil unsere europäischen Freunde das benötigen“.

Ganz ähnlich beschreibt diesen Umstand sein Kollege aus einem anderen Tarifgebiet:

Europa ist da nicht so präsent, wie sich das vielleicht in den Broschüren oder wie man sich das in der Wirklichkeit so vorstellt. Wie gesagt, da sitzt keiner im Hinterzimmer und hat den europäischen Rechner auf, tippt das ein und sagt, geht nicht oder geht. Sondern das ist eher so ein Punkt, wo man sagt, wir müssen uns auch dazu rechtfertigen, wir müssen das einfließen lassen, aber in der praktischen Arbeit habe ich jetzt keinen Ordner in der Tarifrunde 2015, wo ich jetzt Europa stehen habe.

Die Ausführungen der befragten Vertreter der IG Metall lassen also auf einen unaufgelösten Widerspruch zwischen dem nominellen Bekenntnis und der Perzeption der konkreten Umsetzbarkeit schließen.

Weiten wir unseren Blick nun auf weitere Aspekte der Umsetzung jenseits der nackten Lohnprozente aus, relativieren sich die Rechtfertigungen des Scheiterns über den Verweis auf mangelnde Kapazitäten teilweise. So fällt auf, dass die $I G$ Metall ihre Tarifforderungen bis zur Tarifrunde 2013 nicht mit dem Verweis auf die Zielinflation der EZB, sondern auf die unterdurchschnittliche deutsche Inflationsrate begründete (vgl. Abschn. 2). Auch die Entsendung von Tarifsekretären zu Tarifverhandlungen der ausländischen Schwestergewerkschaften, ein wesentlicher Bestandteil der Koordinierungsinitiative, wird nicht praktiziert. Zudem konstatiert IndustriALL (2014, S. 10) mangelnde Zuverlässigkeit bei der Abgabe der Daten durch die nationalen Mitgliedsorganisationen. ${ }^{13}$

Diese Diskrepanz zwischen nominellem Bekenntnis und tarifpolitischer Praxis relativiert wiederum einer der befragten Tarifsekretäre, indem er vor allem den symbolischen Charakter der Koordinierungsinitiativen betont. Ihm zufolge bedeutet

\footnotetext{
${ }^{13}$ In diesem Zusammenhang ist aber anzumerken, dass die Vergleichbarkeit der in die Eucob@n-Datenbank eingespeisten Tarifdaten ohnehin mit einem Fragezeichen zu versehen ist, weil die Abgrenzung der relevanten Tarifbereiche international erheblich variiert.
} 
Lohnkoordinierung nicht die tatsächliche Abstimmung von Lohnerhöhungen (oder zumindest entsprechender Forderungen), sondern eine programmatische Aufforderung dazu. Ein anderer Befragter drückt den Sachverhalt wie folgt aus:

Wenn ich alles nur auf Praxis nehme und sage, die gemeinsame große Idee ist mir egal, das wird auch nicht funktionieren. Aber dieses Spannungsfeld, das macht ja eigentlich das aus meiner Sicht erst so interessant, sozusagen, aus gewissen Gründen, verwehre ich jetzt der Praxis auch etwas. Oder ich mache es dann eben nicht. Oder ich versuche, es noch mal besser zu machen, als ich es heute hinkriege. Aber ich kann nicht nur auf der einen Seite mich bewegen. Ich muss sozusagen das ganze Feld auch mit betrachten.

Auf die Frage, ob ihm so eine in erster Linie symbolische Koordinierung angesichts der unmittelbaren Problemsituation nicht frustrierend erscheine, erläutert ein IndustriALL-Vertreter seine Auffassung von Interessenvertretung als langfristig und kontinuierlich angelegte Verfolgung von Zielen:

You want early retirement at 58 in your sector. And you ask it from the employers. And they don't give it to you. In two years' time, we come back and we want early retirement with 58. And if we don't succeed, we come back and want early retirement at 58 . Until finally we succeed. It is the objective in collective bargaining.

Diesen Ansatz beschreibt er als ,working towards the future and guiding it“: Aktuelle Initiativen zur Lohnkoordinierung dienen demnach der graduellen Etablierung einer in unbestimmter Zukunft nutzbaren europäischen Verhandlungsarena und nicht der Koordinierung der Tarifpolitik in der Gegenwart. Es geht darum, durch das anhaltende Inszenieren entsprechender Bemühungen die eigene Handlungskapazität zu begründen. Das Nichtfunktionieren der Abstimmung ist so zwar weiterhin ein Problem, gemessen an den eigenen Erwartungen aber keines, das es in der unmittelbaren Gegenwart zu lösen gilt.

\section{Eine pragmatistische Interpretation}

Wie lassen sich die in Abschn. 5 dargestellten Verhaltensweisen im Lichte einer Politischen Soziologie der europäischen Gewerkschaftszusammenarbeit interpretieren? Auf Grundlage eines rationalistischen Handlungsmodells, welches die gezielte Verfolgung bewusster Interessen als stabile Verhaltensweise zugrunde legt, erscheint das Nebeneinander von drastisch scheiternder Lohnkoordination und ungebrochener Bekenntnis zu ihr absurd. Warum sollten sich vernünftige Akteure dergestalt verhalten, und das auch noch über mindestens anderthalb Jahrzehnte hinweg? Der Eindruck der Unvernunft schwindet jedoch, so wollen wir nachfolgend aufzeigen, wenn man das rationale Handlungsmodell durch eine soziologisch-aufgeklärte Interpretation ersetzt, die sich an Elementen des Amerikanischen Pragmatismus sowie des Skandinavischen Institutionalismus orientiert. Geht man so vor, erkennt man, dass sich die beteiligten Akteure nicht ,,irrational“, „unvernünftig“ oder „falsch“ verhalten. Vielmehr vollbringen sie vor dem Hintergrund unvereinbarer Umweltan- 
forderungen kreative Leistungen und arbeiten daran, effektive Koordination trotz ihres permanenten Scheiterns als auf die ferne Zukunft gerichtetes Leitbild lebendig zu halten.

Als Vertreter einer skandinavischen Spielart des Soziologischen Institutionalismus betont Nils Brunsson die Tendenz von Organisationen, widersprüchlichen Umwelterwartungen durch die Entkoppelung von Reden und Handeln zu entsprechen. Dass diese Entkoppelung besonders für politische Organisationen als wahrscheinlich gilt (Brunsson 1989, S. 174), lässt keineswegs den automatischen Schluss auf strategische Absicht zu: Was oberflächlich wie systematischer Betrug wirken mag, stellt tatsächlich oft ein (provisorisches) Zwischenergebnis unterschiedlicher, miteinander verbundener Handlungsstränge dar. Diese theoretischen Einsichten korrespondieren in auffälliger Weise mit unseren Beobachtungen im Feld der lohnpolitischen Koordinierungsversuche auf europäischer Ebene. Trotz der in solchen Fällen auftretenden realen Entkoppelungsdynamik beschreiben auch Meyer und Rowan (1977, S. 59) den aufrichtigen Wunsch der beteiligten Akteure, formale Zielsetzungen erreichen zu wollen: „The commitments built up by displays of morale and satisfaction are not simply vacuous affirmations of institutionalized myths".

An dieser Stelle findet sich ein Berührungspunkt zu einem Strang handlungstheoretischer Erwägungen aus dem Bereich des amerikanischen Pragmatismus. Als „philosophy of evolutionary learning“ (Ansell 2011, S. 5) rückt dieser die interaktive Sinnkonstruktion als Sequenz praktischen Problemlösens (Emirbayer und Mische 1998, S. 967 f.) in den Blick: „Handeln wird so nicht teleologisch verstanden, als kontrolliert durch ein außerhalb des Handlungsprozesses festgelegtes Ziel, und auch nicht traditional, als basierend auf nicht hinterfragbaren Routinen" (Beckert 2014, S. 64). Kreatives Problemlösen vollzieht sich aus dieser Sicht, indem Akteure Zwecke und Ziele in der Auseinandersetzung mit spezifischen Gegebenheiten (weiter-)entwickeln oder indem sie, wie Festenstein (zitiert nach Ansell 2016, S. 396) bemerkt, ,define the specific problem that the situation presents and to re-establish in accordance with human purposes the provisional equilibrium which held".

Was als Entkoppelung von Reden und Handeln begonnen haben mag, kann sich im Prozess der andauernden Verhandlung also prinzipiell durchaus als Konvergenzbewegung darstellen. Hierauf sind, zu Recht oder zu Unrecht, die Hoffnungen der an der europäischen Lohnkoordinierung Beteiligten gerichtet. Eine großspurige Ankündigung kann die eine oder den anderen zu Höchstleistungen anspornen. Ungeachtet der Entwicklung realer Kapazitäten - funktionale Lohnkoordination hat, wie wir gesehen haben, institutionelle, organisationale und motivationale Voraussetzungen in den beteiligten Ländern, die durch die europäischen Akteure nicht steuerbar sind -, erscheint es gleichzeitig zumindest denkbar, dass bestimmte Handlungs- beziehungsweise Koordinationsmöglichkeiten erst im Zeitverlauf offenbar und/oder zugänglich werden.

Zwar ist aus einer bloßen Behauptung, auch wenn sie anhaltend geäußert wurde, keinerlei Garantie über die nachholende Verwirklichung der proklamierten Sachverhalte zu erwarten. Auch ist mit Brunsson (1989) durchaus von der Möglichkeit auszugehen, dass Entscheidungsträger anhaltende Reformversprechen zur Rationalisierung politischer Lebenslügen hinzuziehen. Eine solche Lebenslüge kann auch bei den Bekenntnissen zur europäischen Lohnkoordinierung vorliegen. Gleichzeitig ist 
der Etablierung und Umsetzung politischer Positionen als Mobilisierungsprozessen Beckert (2016) zu Folge aber stets ein genuin fiktionales Moment zu unterstellen, welches offen zuerst in seiner sprachlichen Äußerung zutage tritt. Die Entwicklung politischer Positionen und Kapazitäten stellt sich aus dieser Sicht also als ein „creative adjustment“ (Overdevest 2011, S. 539) und „learning through surprise“ (Overdevest und Zeitlin 2012, S. 280) dar.

Mit diesen Erwägungen wird deutlich, wie sich die eigentümlich-widersprüchliche Dynamik, die das Handlungsfeld der europäischen Lohnkoordinierung - trotz anhaltenden Widerspruchs zwischen nomineller Programmatik und Praxis - am Leben hält, auf der Ebene der beteiligten Organisationen rationalisieren kann. Was aber folgt daraus für die Aussichten auf eine effektive europäische Lohnkoordination?

\section{Fazit: Feste Wechselkurse ohne effektive Lohnkoordination}

Der Ausgangspunkt unserer Überlegungen war, dass sich das funktionale Erfordernis effektiver Lohnkoordination mit der unwiderruflichen Fixierung der am Euro teilnehmenden Länder im Jahr 1999 erheblich vergrößert hat (Abschn. 1). Wie unsere Betrachtungen in den Abschn. 2 und 3 zeigten und zudem das Praxisbeispiel aus Abschn. 5 bestätigte, hat sich im Lauf der letzten fünfundzwanzig Jahre und verstärkt seit der Einführung des Euro in der Tat ein transnationales Handlungsfeld verdichtet, dessen Protagonisten sich mit der Koordinierung von Löhnen beschäftigen. Allerdings stehen der effektiven Orientierung an den Imperativen der Lohnkoordination strukturell widrige Umstände entgegen (Abschn. 4 und 5). Hierzu zählen im deutschen Beispiel etwa die zunehmend glaubhaft gewordenen Drohungen mit Standortverlagerungen und die Dezentralisierung des Tarifsystems, die die Orientierung der Lohnpolitik an gesamtwirtschaftlichen oder gar europäischen Imperativen erschwert. Allgemein setzt effektive Lohnkoordination eine Steuerbarkeit der Lohnentwicklungen in den Euro-Teilnehmerländern voraus, die gegenwärtig nicht gegeben ist. Zwar wissen wir nicht, ob sich die Lohnauftriebe im Euroraum noch stärker auseinanderentwickelt hätten, wenn die beteiligten Verbände ihre Bekenntnisse zur Koordinierung gänzlich fallengelassen und sich stattdessen offen zu einer lohnpolitisch betriebenen Standortkonkurrenz bekannt hätten. Die in Abschn. 4 gezeigten Daten lassen aber keine Zweifel an der Interpretation zu, dass eine effektive Lohnkoordination im Euroraum seit 1999 nicht stattgefunden hat.

Im Widerspruch hierzu ist die Lohnkoordinierung aber zum festen Bestandteil der programmatischen Leitbilder von IG Metall und IndustriALL geworden und auch geblieben. Unser Interpretationsvorschlag lautet, diese Diskrepanz nicht als Ausdruck ,,irrationaler“ oder ,falscher“" Handlungsweisen der beteiligten Akteure zu interpretieren. Der Rückgriff auf soziologisch aufgeklärte Handlungstheorien führt uns vielmehr zu der These, dass im Feld der Lohnkoordinierung eine pragmatische Entkoppelung von Reden und Handeln stattgefunden hat (Abschn. 5 und 6).

Diesem Umstand nun mit Zynismus zu begegnen, würde den unvereinbaren Umweltansprüchen, mit denen sich die Akteure im Feld der Lohnkoordinierung konfrontiert sehen, nicht gerecht. Eine faire Beurteilung des Status quo der Lohnkoordinierung muss unseres Erachtens die genuine Zukunftsorientierung politischer Mobili- 
sierung in Rechnung stellen. Die beteiligten Akteure sind sich des durchgreifenden Scheiterns effektiver Lohnkoordination sehr wohl bewusst. Ihre Aufmerksamkeit ist auf die Aufrechterhaltung der Möglichkeit einer in mittel- bis langfristiger $\mathrm{Zu}$ kunft stattfindenden Lohnkoordination gerichtet. Das Handlungsfeld, in dem diese zukunftsgerichteten Handlungen stattfinden, ist das gegenwärtige Feld der Lohnkoordinierung.

Was im Sinne Brunssons (1989) als Entkoppelung von Reden und Handeln zum Zweck einer Harmonisierung konträrer Erwartungshaltungen erscheinen mag, stellt aus dieser Sicht eine kreative Anpassung an widrige Bedingungen dar: Um ihre politische Handlungsfähigkeit zu erhalten, deuten die Akteure den Zweck der Lohnkoordinierung um. Dies ist aus sich selbst heraus weder ausschließlich positiv noch negativ zu beurteilen. Eine positive Interpretation würde in Rechnung stellen, dass ein rhetorisches Bekenntnis zum Projekt der Lohnkoordinierung auch unter widrigen Bedingungen einen ersten wichtigen Schritt in diese Richtung darstellen könnte, ,in the sense that novel institutions do not emerge ex nihilo" (Sabel und Zeitlin 2012, S. 423). Dass jemand etwas tun will und soll, heißt zwar nicht, dass er es auch kann - aber eben genauso wenig, dass er es nie können wird. Der gegenwärtige Stand der Dinge lässt aber durchaus auch eine skeptischere Deutung zu. Die Aufrechterhaltung der normativen Programmatik kann in ein ritualisiertes Bekenntnis ohne Aussicht auf Handlungskonsequenz abrutschen, das praktisch nicht mehr bewirkt, als eine zweifelhafte Außendarstellung aufrechtzuerhalten.

Vor dem Hintergrund dieser divergenten Deutungsmöglichkeiten der Zukunftsaussichten europäischer Lohnkoordinierung erscheint der Titel, den wir diesem Beitrag gegeben haben, rechtfertigungsbedürftig: „Gab es nicht, gibt es nicht, wird es nicht geben“. Die Fragestellung, auf die wir diese Antwort geben, lautete allerdings nicht, ob es eine transnationale Lohnkoordination in ferner Zukunft einmal geben könnte, sondern vielmehr, ob es eine solche zur Stabilisierung des Euro geben wird. Die Eurokrise aber existiert heute und ihre Lösung würde eine höchst effektive Lohnkoordination in naher Zukunft erfordern.

Auf Grundlage aller zur Verfügung stehenden Informationen wäre es verantwortungslos, hier falsche Hoffnungen zu wecken. Wer solche Hoffnungen dennoch schürt, verwechselt das aus guten Gründen erfolgende Festhalten an auf die mittelbis langfristige Zukunft gerichteten Programmatiken mit den heute und kurzfristig vorhandenen Handlungsoptionen. Wenn also beispielsweise Busch et al. (2016, S. 70) in ihrer Streitschrift für eine Reform der Eurozone unter Beibehaltung des Euro eine ,europäische Koordinierung der nationalen Tarifpolitiken“" zu Recht als eines der „Hauptinstrumente“ für ein friktionsloses Funktionieren der Währungsunion angeben, wird man antworten müssen, dass dem Euro genau diese Funktionsvoraussetzung fehlt und in der absehbaren, also kurz- bis mittelfristigen Zukunft auch weiter fehlen wird. Der Währungsunion fehlt damit eine, wenn nicht sogar die entscheidende Bestandsvoraussetzung.

Danksagung Für wertvolle Hinweise danken wir Hans-Jürgen Bieling, Wolfgang Streeck und zwei anonymen Gutachtern.

Open Access Dieser Artikel wird unter der Creative Commons Namensnennung 4.0 International Lizenz (http://creativecommons.org/licenses/by/4.0/deed.de) veröffentlicht, welche die Nutzung, Vervielfäl- 
tigung, Bearbeitung, Verbreitung und Wiedergabe in jeglichem Medium und Format erlaubt, sofern Sie den/die ursprünglichen Autor(en) und die Quelle ordnungsgemäß nennen, einen Link zur Creative Commons Lizenz beifügen und angeben, ob Änderungen vorgenommen wurden.

Funding Open access funding provided by Max Planck Society.

\section{Literatur}

Ansell, Chris. 2011. Pragmatist democracy: Evolutionary learning as public philosophy. Oxford: Oxford University Press.

Ansell, Chris. 2016. Pragmatism. In Handbook on theories of governance, Hrsg. Chris Ansell und Jacob Torfing, 392-402. Cheltenham: Elgar.

Baccaro, Lucio, und Jonas Pontusson. 2016. Rethinking comparative political economy: The Growth models perspective. Politics and Society 44:175-207.

Beckert, Jens. 2014. Capitalist dynamics. Fictional expectations and the openness of the future. MPIfG Discussion Paper 14/7. Köln: Max-Planck-Institut für Gesellschaftsforschung.

Beckert, Jens. 2016. Imagined futures: Fictional expectations and capitalist dynamics. Boston: Harvard University Press.

Bispinck, Rainer, und Thorsten Schulten. 2006. Perspektiven einer gesamtwirtschaftlich orientierten Lohnpolitik. In Kein bisschen leise: 60 Jahre WSI, Hrsg. Claus Schäfer und Hartmut Seifert, 253-270. Hamburg: VSA.

Brunsson, Nils. 1989. The organization of hypocrisy: Talk, decisions and actions in organizations. Hoboken: John Wiley \& Sons.

Busch, Klaus, Axel Troost, Gesine Schwan, Frank Bsirske, Joachim Bischoff, Mechthild Schrooten und Harald Wolf. 2016. Europa geht auch solidarisch! Streitschrift für eine andere Europäische Union. Hamburg: VSA.

Collignon, Stefan. 2009. The failure of the macroeconomic dialogue on wages (and how to fix it). In The sinews of European peace - reconstituting the democratic legitimacy of the socio-economic constitution of the European Union, Hrsg. Raúl Letelier und Agustín José Menéndez, 427-469. ARENA Report No 7/09 \& RECON Report No 10. Oslo. http://www.stefancollignon.de/PDF/RECONreport0910_ chap10.pdf.

Deppe, Frank. 2012. Gewerkschaften in der Großen Transformation: Von den 1970er Jahren bis heute. Köln: PapyRossa.

Emirbayer, Mustafa, und Ann Mische. 1998. What is agency? American Journal of Sociology 103:962-1023

Erne, Roland. 2008. European Unions: Labors quest for a transnational democracy. Ithaca: Cornell University Press.

Erne, Roland. 2013. Coordinating collective wage bargaining: A way of transnational solidarity in Europe? Trade unions and global restructuring, 11. Oktober. http://andreasbieler.blogspot.de/2013/10/ coordinating-collective-wage-bargaining.html.

Esping-Andersen, Gøsta. 1990. The three worlds of welfare capitalism. Cambridge: Polity.

Flassbeck, Heiner, und Costas Lapavitsas. 2015. Against the troika: Crisis and austerity in the Eurozone. London: Verso.

Ghali, Khalifa H. 1999. Wage growth and the inflation process: A multivariate cointegration analysis. Journal of Money, Credit and Banking 31:417-431.

Glassner, Vera, und Susanne Pernicka. 2014a. Transnational trade union strategies towards European wage policy: A neo-institutional framework. European Journal of Industrial Relations 20:1-18.

Glassner, Vera, und Susanne Pernicka. 2014b. Transnationale Strategien der Gewerkschaften im europäischen Metallsektor: Ansätze zur Europäisierung der Lohnpolitik. Industrielle Beziehungen 21:277-299.

Glassner, Vera, und Philippe Pochet. 2011. Why trade unions seek to coordinate wages and collective bargaining in the Eurozone. ETUI Working Paper 3/2011. Brüssel: European Trade Union Institute.

Glassner, Vera, und Andrew Watt. 2010. The current crisis reveals oth: The importance and the limitations of the transnational coordination of collective bargaining policies. http://www.social-europe.eu/ 2010/07/the-current-crisis-reveals-both-the-importance-and-the-limitations-of-the-transnationalcoordination-of-collective-bargaining-policies/.

Glassner, Vera. 2009. Two paths towards Europeanisation: The institutional and economic determinants of the transnationalization of collective bargaining on the European sector level. Diss. Wien: Universität. 
Haipeter, Thomas. 2011. Tarifabweichungen, Betriebsräte und Gewerkschaften - Modernisierungschancen in lokalen Konflikten. In Gewerkschaftliche Modernisierung, Hrsg. Thomas Haipeter und Klaus Dörre, 31-60. Wiesbaden: VS Verlag für Sozialwissenschaften.

Hall, Peter A. 2014. Varieties of capitalism and the Euro crisis. German Politics 21:355-371.

Hall, Peter A., und David Soskice. 2001. An introduction to varieties of capitalism. In Varieties of capitalism: Institutional foundations of comparative advantage, Hrsg. Peter A. Hall und David Soskice, 1-68. Oxford: Oxford University Press.

Hancké, Bob. 2013. Unions, Central Banks and EMU: Labour market institutions and monetary integration in Europe. Oxford: Oxford University Press.

Hassel, Anke, und Christof Schiller. 2010. Der Fall Hartz IV: Wie es zur Agenda 2010 kam und wie es weitergeht. Frankfurt a.M.: Campus.

Henning, Klaus. 2013. Europäische Integration und Gewerkschaften: Der EMB zwischen Interessenvertretung und transnationaler Solidarität. Wiesbaden: Springer VS.

Hoffmann, Jürgen, und Reiner Hoffmann. 1997. Globalization-Risks and opportunities for European labor policy. ETUI-Discussion and Working Paper. Brüssel: European Trade Union Institute.

Höpner, Martin. 2016. Das deutsche Lohnregime und der Merkantilismus. Makroskop, 27. Mai. http:// www.mpifg.de/people/mh/paper/Hoepner2016_Das_deutsch_Lohnregime_und_der_Merkantilismus. pdf.

Höpner, Martin, und Mark Lutter. 2018. The diversity of wage regimes: Why the Eurozone is too heterogeneous for the Euro. European Political Science Review 10:71-96.

Höpner, Martin, und Alexander Spielau. 2016. Besser als der Euro? Das Europäische Währungssystem (EWS), 1979-1998. Berliner Journal für Soziologie 26:273-296.

IndustriALL. 2012. Politische Entschließung: Vom Gründungskongress der IndustriAll European Trade Union am 16.05.2012 in Brüssel verabschiedet. Ind. 2/2012. Brüssel: International Trade Union House.

IndustriALL. 2014. Unsere Zukunft verhandeln! Gewerkschaftliche Strategien in Zeiten der Wirtschaftskrise. Dokument zur Tarif- und sozialpolitischen Konferenz von IndustriALL, 12.-13. Juni. Wien.

IMF (International Monetary Fund). 2015. World economic outlook: Adjusting to lower commodity prices. Washington, DC: International Monetary Fund.

Johnston, Alison. 2009. Wage policy in Austria and the Netherlands under EMU: A change in performance or the continuation of the status-quo? ETUI Working Paper 7/2009. Brüssel: European Trade Union Institute.

Johnston, Alison, und Aidan Regan. 2014. European integration and the incompatibility of different varieties of capitalism: Problems with institutional divergence in a monetary union. MPIfG Discussion Paper 14/15. Köln: Max-Planck-Institut für Gesellschaftsforschung.

Lehndorff, Steffen. 2014. Der eingebildete Gesunde: Die Neue Karriere des ,Modells Deutschland“. In Die Spaltende Integration: Der Triumph gescheiterter Ideen in Europa - revisited. Zehn Länderstudien, Hrsg. Steffen Lehndorff, 131-159. Hamburg: VSA.

Marginson, Paul. 2014. Coordinated bargaining in Europe: From incremental corrosion to frontal assault? European Journal of Industrial Relations 20:37-51.

Marginson, Paul, und Franz Traxler. 2005. After enlargement: Preconditions and prospects for bargaining coordination. Transfer 11:423-438.

Marginson, Paul, Maarten Keune und Dorothee Bohle. 2014. Negotiating the effects of uncertainty? The governance capacity of collective bargaining under pressure. Transfer 20:37-51.

IG Metall. 2005. Europäische Koordinierung nationaler Tarifpolitik. Frankfurt a.M.: IG Metall.

IG Metall. 2009. Für ein solidarisch erneuertes Europa: Europapolitisches Memorandum der IG Metall. Frankfurt a.M.: IG Metall.

Meyer, John W., und Brian Rowan. 1977. Institutionalized organizations: Formal structure as myth and ceremony. American Journal of Sociology 83:340-363.

Overdevest, Christine. 2011. Towards a more pragmatic sociology of markets. Theory and Society 40:533-552.

Overdevest, Christine, und Jonathan Zeitlin. 2012. Assembling an experimentalist regime: Transnational governance interactions in the forest sector. Regulation and Governance 8:22-48.

Pen, Jan. 1963. Europäische Lohnpolitik. Gewerkschaftliche Monatshefte 63:581-585.

Perez, Sofia A. 2002. Monetary union and wage bargaining institutions in the EU: Extrapolating from some member state experiences. Comparative Political Studies 35:1189-1227.

Pulignano, Valeria. 2010. Trade unions and transnational regulation in Europe: Developments and Limitations. Employee Relations 32:574-589. 
Rüb, Stefan. 2009. Die Transnationalisierung der Gewerkschaften: Eine empirische Untersuchung am Beispiel der IG Metall. Berlin: edition sigma.

Sabel, Charles F., und Zeitlin, Jonathan. 2012. Experimentalism in the EU: Common ground and persistent differences. Regulation \& Governance 6:410-426.

Scharpf, Fritz W. 1987. Sozialdemokratische Krisenpolitik in Europa. Frankfurt a.M.: Campus.

Schroeder, Wolfgang, und Rainer Weinert. 2003. Europäische Tarifpolitik: Ein neues Politikfeld. In Die Gewerkschaften in Politik und Gesellschaft der Bundesrepublik Deutschland: Ein Handbuch, Hrsg. Wolfgang Schröder und Bernhard Wessels, 565-587. Wiesbaden: Westdeutscher Verlag.

Schroeder, Wolfgang, und Rainer Weinert. 2004. Designing institutions in European industrial relations: A strong commission versus weak trade unions? European Journal of Industrial Relations 10:199-217.

Schulten, Thomas. 2001. Solidarische Lohnpolitik in Europa: Ansätze und Perspektiven einer Europäisierung gewerkschaftlicher Lohnpolitik. WSI Diskussionspapier 92. Düsseldorf: Hans-Böckler-Stiftung.

Schulten, Thorsten. 2004. Solidarische Lohnpolitik in Europa: Zur Politischen Ökonomie der Gewerkschaften. Hamburg: VSA.

Schulten, Thorsten, und Reinhard Bispinck. Hrsg. 1999. Tarifpolitik unter dem Euro. Perspektiven einer europäischen Koordinierung: Das Beispiel Metallindustrie. Hamburg: VSA.

Schulten, Thorsten, und Torsten Müller. 2013. Ein neuer europäischer Interventionismus? Die Auswirkungen des neuen Systems der europäischen Economic Governance auf Löhne und Tarifpolitik. Wirtschaft und Gesellschaft 39:291-320.

Seeliger, Martin. 2017. Die soziale Konstruktion organisierter Interessen: Gewerkschaftliche Positionsbildung im Zuge der europäischen Integration. Frankfurt a. M.: Campus.

Sinn, Hans-Werner. 2014. The Euro trap: On bursting bubbles, budgets, and beliefs. Oxford: Oxford University Press.

Sterkel, Gabriele, und Jörg Wiedemuth. 2011. Neue Solidarische Tarifpolitik. Sozialismus 38:26-32.

Sterkel, Gabriele, Thorsten Schulten und Jörg Wiedemuth. Hrsg. 2004. Autonomie im Laufstall? Gewerkschaftliche Lohnpolitik in Euroland? Hamburg: VSA.

Storm, Servaas, und C. W. M. Naastepad. 2015. Myths, mix-ups and mishandlings: What caused the Eurozone crisis? Konferenzpapier. Annual conference of the Institute for New Economic Thinking. Paris: Institute for New Economic Thinking.

Streeck, Wolfgang. 1994. Staat und Verbände: Neue Fragen. Neue Antworten? In Staat und Verbände, Hrsg. Wolfgang Streeck, Bd. 25 Politische Vierteljahresschrift, Sonderhefte, 7-34. Opladen: Westdeutscher Verlag.

Thelen, Kathleeen. 2014. Varieties of liberalization and the new politics of social solidarity. Cambridge: Cambridge University Press.

Trampusch, Christine. 2009. Der erschöpfte Sozialstaat: Transformation eines Politikfeldes. Frankfurt a. M.: Campus.

Traxler, Franz. 2000. Das Tarifverhandlungssystem in der Wirtschafts- und Währungsunion: Von nationalen zu supranationalen Institutionen? In Die Europäisierung nationaler Gesellschaften, Sonderheft 40 der Kölner Zeitschrift für Soziologie und Sozialpsychologie, Hrsg. Maurizio Bach, 93-11. Opladen: Westdeutscher Verlag.

Traxler, Franz, und Bernd Brandl. 2009. Towards Europeanization of wage policy: Germany and the nordic countries. European Union Politics 10:177-201.

Traxler, Franz, Bernd Brandl, Vera Glassner und Alice Ludvig. 2008. Can cross-border bargaining coordination work? Analytical reflections and evidence from the metal industry in Germany and Austria. European Journal of Industrial Relations 14:217-237.

Weinert, Rainer. 2001. Zur Zwangseuropäisierung nationaler Gewerkschaften. Soziale Welt 52:323-339.

Wyplosz, Charles. 2013. Eurozone crisis: It's about demand, not competitiveness. Geneva: The Graduate Institute.

Martin Höpner Prof. Dr., Politikwissenschaftler und Forschungsgruppenleiter am Kölner Max-PlanckInstitut für Gesellschaftsforschung (MPIfG). Forschungsgebiet: Schwerpunktgebiet ist die Vergleichende Politische Ökonomie. Letzte Veröffentlichungen: Der integrationistische Fehlschluss. Leviathan 43, 2015; Endlich Subsidiarität? Die parlamentarische Subsidiaritätskontrolle am Beispiel von „Monti II“. Politische Vierteljahresschrift 57, 2016 (m. L. Ehret). 
Martin Seeliger Dr., wissenschaftlicher Mitarbeiter im Seminar für Soziologie der Europa-Universität Flensburg. Forschungsbereiche: (Internationale) Arbeitsbeziehungen und Tarifpolitik, politische Soziologie, soziale Ungleichheit, Organisationsforschung, Cultural Studies. Letzte Veröffentlichungen: Die soziale Konstruktion organisierter Interessen. Gewerkschaftliche Positionsbildung im Zuge der europäischen Integration. Frankfurt a. M. 2017; Trade unions under the pressure of European integration. A question of optimism and pessimism? London 2018 (i.E. mit J. Kiess als Hrsg.); Internationale Gewerkschaftspolitik im Prozess der europäischen Integration. Frankfurt a. M. 2018 (i.E., mit J. Kiess als Hrsg.); Deutscher Gangstarap II. Popkultur als Kampf um Integration und soziale Ungleichheit. Bielefeld 2017 (mit M. Dietrich als Hrsg.); Probleme transnationaler Lohnkoordination zur Stabilisierung des Euro. Eine soziologischpolitökonomische Perspektive. In: Beyer, Jürgen; Trampusch, Christine (Hrsg.): Finanzmarkt, Demokratie und Gesellschaft. Sonderheft der Kölner Zeitschrift für Soziologie und Sozialpsychologie (i. E., mit M. Höpner); Why do (some) European trade unions reject minimum wage regulation? Trade union politics between Integrationism and Institutional Nationalism. Culture, Practice and Europeanization 3, 2018 ; Warum die EU aus gewerkschaftlicher Sicht keine Solidargemeinschaft darstellt. Leviathan 45, 2017. 